\title{
Violência entre pares, clima escolar e contextos de desenvolvimento: suas implicações no bem-estar
}

\author{
Peer violence, school environment and developmental contexts: \\ its effects on well-being
}

\author{
Stefania Carneiro de Alcantara ${ }^{1}$ \\ Mònica González-Carrasco ${ }^{1}$ \\ Carme Montserrat ${ }^{1}$ \\ Ferran Casas ${ }^{1}$ \\ Ferran Viñas-Poch ${ }^{1}$ \\ Desirée Pereira de Abreu ${ }^{1}$
}

\footnotetext{
${ }^{1}$ Instituto de Investigaciones sobre Calidad de Vida, Facultad de Educación y Psicología, Universitat de Girona. Plaça de Sant Domènec 3. 17004 Giron Espanha.stefaniacarneiro@ yahoo.com.br
}

\begin{abstract}
The scope of this study is to analyze the implications of peer violence in the school context, the school environment and the perceived developmental contexts on the subjective well-being of children and adolescents. The sample is comprised of 910 students in Years 6 and 7 of primary school in 27 urban and rural public and private schools in Ceará. It is a quantitative cross-sectional study and the following tools were used: scales measuring victimization and aggression among peers and the school environment; three indices on perceptions of developmental contexts (home, school, neighborhood); and three well-being scales (Students' Life Satisfaction Scale, Personal Well-being Index and Single item on Overall Life Satisfaction). Data were analyzed using multivariate variance analysis, with a significance level below 0, 01. Results indicate that bullying, alone or in interaction with the variables analyzed, is the variable that has the greatest impact on the well-being of the population studied, whereby those involved in bullying have the lowest well-being averages. Low levels of perception regarding the contexts of home, school and neighborhood and the school environment, as well as attending a public school and/or living in rural areas, are all also associated with low subjective well-being. Key words Violence, Bullying, Quality of life, Child, Adolescent
\end{abstract}

Resumo $O$ objetivo desta pesquisa é analisar as implicações da violência entre pares no contexto escolar, do clima escolar e da percepção dos contextos de desenvolvimento no bem-estar subjetivo de crianças e adolescentes. A amostra é composta por 910 estudantes do $6^{\circ}$ e $7^{\circ}$ ano do ensino fundamental, de 27 escolas, públicas e privadas, urbanas e rurais do Ceará. Trata-se de um estudo transversal quantitativo que utilizou os instrumentos escala de vitimização e agressão entre pares e de clima escolar; três indices de percepção dos contextos de desenvolvimento (casa, escola, bairro); três escalas de bem-estar (satisfação com a vida para estudantes, indice de bem-estar pessoal $e$ item único de satisfação com a vida) para coleta de dados. Os dados passaram por análise de variância multivariada com nível de significância não superior a 0,01. Os resultados indicam que a tipologia bullying, isoladamente ou em interação com as variáveis analisadas, é a que mais impacto exerce sobre o bem-estar da população estudada, sendo os envolvidos os que apresentam as menores médias de bem-estar. Níveis baixos de percepção dos contextos casa, escola, bairro, e do clima escolar, bem como estudar em escola pública e/ou morar em zona rural, estão igualmente associados a bem-estar subjetivo baixo.

Palavras-chave Violência, Bullying, Qualidade de vida, Criança, Adolescente 


\section{Introdução}

A violência entre pares no contexto escolar (bullying) tem sido considerada fator de risco ao desenvolvimento saudável ${ }^{1}$, afetando a saúde, a qualidade de vida e o bem-estar subjetivo, físico e social de crianças e adolescentes ${ }^{2}$. Trata-se de condutas agressivas com diferentes níveis e tipos de violência (física, verbal, e/ou psicológica), intencional, repetitiva ao longo do tempo; sem motivação aparente, que ocorrem entre pares e em uma relação desigual de poder e força. Os envolvidos ocupam papéis de agressores, vítimas ou vítimas-agressoras ${ }^{3}$. Entre os principais fatores de risco associados a literatura indica: baixa autoestima $^{4-7}$, depressão $0^{5-7}$, ansiedade ${ }^{6}$, estresse ${ }^{7}$. Além disso, fatores como a percepção do clima familiar $^{7,8}$, escolar ${ }^{7-10}$ e comunitário ${ }^{7}$ também estão associados ao fenômeno, sendo a baixa percepção associada negativamente (fatores de risco) e a alta positivamente (fatores de proteção).

Entendida enquanto um fenômeno multidimensional, a violência entre pares no contexto escolar resulta de complexas interações entre crianças e adolescentes e seus ambientes sociais ${ }^{8,11}$. No caso de crianças e adolescentes, casa, escola e comunidade se configuram como os principais contextos onde vivem e se desenvolvem sendo necessário estudá-los desde a perspectiva destes atores, se pretendemos entender seus modos de vida e conhecer os fatores de risco e proteção presentes ${ }^{12}$.

Igualmente relevante é conhecer o conjunto de percepções que crianças e adolescentes têm acerca de sua escola (clima escolar) ${ }^{13}$ e que influi em seu comportamento, e, por conseguinte, em seu desenvolvimento e saúde.

Neste estudo, partimos do enfoque ampliado de saúde enquanto estado de bem-estar físico, psicológico e social, e não somente ausência de doenças; o que pressupõe em seu processo de construção e promoção os determinantes de saúde, presentes nas diferentes formas de organização social, que determinam modos de vida e o que se entende por qualidade de vida ${ }^{14}$. Na perspectiva das ciências sociais, qualidade de vida é entendida como função das condições materiais (bem-estar objetivo) e das condições psicossociais (bem-estar subjetivo), baseado este último no conjunto de percepções, avaliações e aspirações que têm as pessoas acerca de sua própria vida em geral (satisfação vital) e de seus distintos âmbitos a partir da cultura e sistema de valores nos quais elas vivem e em relação aos seus objetivos, expectativas, experiências, etc. O bem-estar resulta da interinfluência entre os aspectos internos ou psicológicos e suas interações externas com outras pessoas e com o ambiente ${ }^{12}$.

No estudo do bem-estar existem dois tipos de instrumentos: os que assumem que a medição deve se basear em perguntas gerais sobre a satisfação com a vida (OLS); e os que consideram que a satisfação global está relacionada com a acumulação de satisfações com os distintos âmbitos da vida $^{12}$ (PWI-SC, SLSS).

Com o intuito de avançar na compreensão dos fatores de risco e proteção que podem incidir no bem-estar, pesquisas recentes de âmbito internacional têm focalizado a atenção ao estudo de variáveis presentes nos distintos contextos de desenvolvimento como: família, escola e comunidade ${ }^{15}$; sentimento de comunidade ${ }^{16}$; integração comunitária ${ }^{8}$; participação familiar ${ }^{17}$; crises familiares ${ }^{18}$; clima escolar e vitimização ${ }^{10}$; climas escolar, familiar, comunitário e violência entre iguais $^{7,8}$; violência entre pares na escola ${ }^{1,5,6,19}$. No contexto escolar, destacam-se os estudos sobre o impacto da violência entre pares no bem-estar subjetivo e os do clima escolar como elemento relevante para compreensão tanto do bem-estar, como da violência entre iguais ${ }^{7,8,10}$. Sendo notório o crescente interesse mundial sobre o tema do bem-estar, inclusive em estudos transculturais ${ }^{2,20}$.

No Brasil, contudo, ainda são escassos os estudos das condições psicossociais (e em específico, do bem-estar subjetivo) que considerem crianças e adolescentes como sujeitos de pesquisa ${ }^{21}$. A temática do bem-estar subjetivo no nordeste do Brasil é relativamente nova, não existindo estudos anteriores com amostras similares em que se tenham analisado o bem-estar desta população.

Buscando preencher a lacuna existente, este estudo tem como objetivo geral analisar as implicações da violência entre pares no contexto escolar, do clima escolar e da percepção dos contextos de desenvolvimento no bem-estar subjetivo dos participantes.

\section{Método}

Trata-se de um estudo transversal, quantitativo, realizado com crianças e adolescentes matriculados em escolas do Ensino Fundamental do Ceará, localizadas em um raio máximo de $100 \mathrm{~km}$ da capital, Fortaleza, e que tinham mais de 160 alunos matriculados em cada série. Dada as limitações orçamentárias e de tempo disponíveis, não se há pretendido obter uma amostra representativa de todo o Estado, mas que refletisse a diversidade da 
população estudada, seguindo o procedimento de amostragem de territórios amplos utilizados no projeto internacional Children's Worlds (www. isciweb.org), do qual se há utilizado também uma parte do questionário. Para obter a amostra utilizou-se a amostragem por conglomerados em duas etapas, selecionando-se primeiro a escola e em seguida o ano escolar $\left(6^{\circ} / 7^{\circ}\right)$. De um universo de 3.887 escolas (3.135 públicas) e (752 privadas) de Ensino Fundamental do Ceará, foram selecionadas, por sorteio, 27 escolas, de 7 municípios do Estado, das quais 13 eram públicas e urbanas, 8 públicas e rurais, 6 privadas e urbanas. A quantidade de escolas urbanas e rurais foi proporcional à distribuição das escolas existentes no Ceará em 2013, onde 2.140 escolas eram urbanas e 1.747 rurais.

\section{Participantes e amostra}

Nas 27 escolas selecionadas havia 3394 alunos matriculados no $6^{\circ}$ e $7^{\circ}$ anos. No dia da administração do questionário bem-estar estavam presentes 2546 alunos, dos quais 1100 apresentaram os Termos de Consentimento e de Assentimento Livre e Esclarecido assinados e preencheram o questionário. Quando da aplicação do questionário bullying e fatores psicossociais 190 alunos não estavam presentes, sendo considerado perdas $(17,27 \%)$. Por conseguinte, a amostra obtida é composta por 910 alunos, 436 meninos (47,9\%) e 474 meninas (52,1\%), com idades de 10 a 16 anos: média $(\mathrm{M})=11,90$ e desvio padrão $(\mathrm{DP})=$ 1,21 , dos quais $(47,6 \%)$ cursavam o $6^{\circ}$ ano e 477 $(52,4 \%)$ o $7^{\circ}$ ano; $763(83,8 \%)$ alunos de escolas públicas; 147 (16,2\%) de privadas; 651 (71,5\%) moravam e estudam em zonas urbanas e 259 $(28,5 \%)$ em zonas rurais do Ceará.

\section{Coleta de dados}

Os questionários foram aplicados de fevereiro a junho de 2014, nas escolas, no horário escolar, em dois dias em cada escola, com duração de 50 minutos, em grupos de 15 a 30 alunos, separados por ano escolar e na presença de duas pesquisadoras responsáveis.

\section{Instrumentos e variáveis}

A coleta de dados se realizou mediante dois questionário de perguntas fechadas: o primeiro baseado no instrumento utilizado no projeto internacional Children's Worlds, versão brasileira validada $^{22}$, composto por dois blocos de pergun- tas, a saber, situação socioeconômica, bem-estar e satisfação com diferentes âmbitos da vida; e o segundo composto por distintas escalas psicométricas de interesse específico para este estudo. Ambos os questionários foram objeto de estudo piloto prévio com 80 estudantes dos mesmos cursos $\left(6^{\circ} / 7^{\circ}\right)$, em quatro escolas, para assegurar a correta compreensão de seu conjunto.

Considerando que pesquisas anteriores têm mostrado que as escalas de bem-estar subjetivo de "livres contexto" e as baseadas em âmbitos da vida não capturam os mesmos aspectos do bem -estar e mostram diferentes níveis de sensibilidade aos aspectos contextuais ${ }^{12}$, igual que no projeto Children's Worlds, utilizamos neste estudo as versões brasileira validadas ${ }^{22,23}$ de três escalas psicométricas: Escala de item único de Satisfação Global com a Vida (Overall Life Satisfaction -OLS ${ }^{24}$, Índice de Bem-estar Pessoal (Personal Well-Being Index - School Children-PWI-SC) ${ }^{25} \mathrm{e}$ Escala de Satisfação com a Vida para Estudantes (Students' Life Satisfaction Scale-SLSS) ${ }^{26}$. Todas estas escalas são unidimensionais e o valor global da PWI-SC e da SLSS se calcula por simples soma aritmética de seus itens.

A escala de item único de Satisfação Global com a Vida-OLS, avalia a satisfação geral com a vida do participante utilizando uma escala de 11 pontos ( $0=$ nada satisfeito e $10=$ totalmente satisfeito):o quanto satisfeito você estar com toda a sua vida em geral.

O Índice de Bem-estar Pessoal-PWI-SC, composto por 7 itens, avalia a satisfação do participante com os âmbitos de sua vida: nível de vida, saúde, realizações, relações (comunitárias e pessoais), segurança (futura e pessoal), em uma escala de 11 pontos $(0=$ nada satisfeito e $10=$ totalmente satisfeito). Os itens trabalhados foram: $o$ quanto você estar satisfeito com... todas as coisas que você tem, sua saúde, seu relacionamento com as pessoas em geral, o seguro que você se sente, fazer coisas fora de casa, o que pode acontecer com você no futuro, etc. Consistência interna encontrada neste estudo: alfa de Cronbach $=0,80$.

E a escala de Satisfação com a Vida para Estudantes-SLSS, composta por 5 itens, avalia o grau de satisfação com a vida: minha vida está indo bem, minha vida está indo como eu quero, eu tenho uma vida boa, as coisas da minha vida são excelentes, etc, em uma escala Likert de 5 pontos (de nada de acordo a muito de acordo). Consistência interna encontrada neste estudo: alfa de Cronbach $=0,88$.

Para medir a percepção dos participantes em relação aos seus contextos de desenvolvimento, 
foram selecionados do instrumento Children's Worlds-International Survey of Children's Well -Being ${ }^{12}$, versão brasileira validada ${ }^{27}$, itens de: Percepção dos Contextos de Desenvolvimento casa (meus pais me tratam bem, meus pais me escutam e levam em conta o que eu falo, eu me sino segura em casa); escola (meus professores me tratam bem, meus professores me escutam e levam em conta o que eu falo, eu me sinto seguro na escola, eu gosto de ir a escola); bairro (eu me sinto seguro quando ando pelas ruas do bairro onde vivo, no meu bairro há lugares suficientes para brincar e me divertir). Os itens são avaliados em uma escala de 5 pontos (de nada de acordo a muito de acordo). Consistências interna encontradas neste estudo: alfa de Cronbach $=0,79$ (Contexto de Desenvolvimento Casa 0,73; Contexto de Desenvolvimento Escola 0,75; Contexto de Desenvolvimento Bairro 0,59). Cada um dos três índices se calcula mediante a soma aritmética dos valores de seus itens.

Para medir a percepção dos alunos sobre o clima escolar, foi utilizada a versão brasileira validada ${ }^{13}$ da Escala de Clima Escolar (Questionário de Clima Escolar) ${ }^{28}$. Instrumento composto em sua forma original, de 22 itens avaliados em escala de 6 pontos $(1=$ discordo totalmente e $6=$ concordo totalmente), e cinco fatores: ambiente físico, pedagógico e social (avalia as condições gerais da escola e para a aprendizagem); regras (aceitação); relações interpessoais (ambiente relacional) uso do tempo (ocupação do horário escolar); indisciplina (existência de indisciplina). Ao considerar-se o alfa da escala se fossem excluídos itens, verificase que a supressão dos itens com conotação negativa aumentaria a consistência interna do instrumento de forma expressiva (alfa inicial antes da supressão Cronbach $=0,84)$. Assim, optou-se pela supressão dos itens: os recreios são pequenos, os alunos têm uma grande carga horária (tempo); na escola há muita indisciplina, os alunos contribuem para um mau ambiente na escola (indisciplina); tenho dificuldade em me relacionar com os professores, e, por conseguinte, a supressão dos fatores tempo e indisciplina.

Os itens trabalhados foram: eu contribuo para um bom ambiente de aprendizagem na escola, o relacionamento entre professores e alunos é bom, os pais dos alunos têm um bom relacionamento com os professores, as regras existentes na escola são bem aceitas pelos alunos, a direção da escola tem bom relacionamento com os alunos, gosto do ambiente da escola, etc. Consistências interna encontradas neste estudo: alfa de Cronbach $=0,91$ (Ambiente físico, social, pedagógico 0,86; Regras 0,73; Relacionamento Interpessoal 0,76$)$. O valor de cada fator se calcula pela soma aritmética dos valores de seus itens.

A partir de agora nos referiremos: a) Percepção de Contexto de Desenvolvimento Casa, Bairro, Escola, como Contexto-Casa, Contexto-Bairro, Contexto-Escola; b) Ambiente Físico, Social e Pedagógico da Escola, como Ambiente-Escolar; c) Relações Interpessoais na escola, como Relacionamento-Escolar.

E para medir os comportamentos de vitimização e agressão foi utilizada a versão brasileira validada $^{29}$ da Escala de Vitimização e Agressão entre Pares-EVAP- (The Aggression Scale) ${ }^{30}$. Instrumento com 18 itens agrupados nas dimensões: agressão direta físicas e verbais (eu dei um empurrão, soquei elou chutei colegas, eu ameacei bater ou fiz outro tipo de ameaça contra colegas); agressão relacional (eu disse coisas sobre colegas para fazer os outros rirem, eu excluí colegas de grupos ou brincadeiras, eu encorajei e incentivei colegas brigarem); vitimização (eu fui empurrado, socado ou chutado, colegas colocaram apelidos em mim que eu não gostei, colegas me excluiram de grupos ou de brincadeiras), avaliados em uma escala Likert de 5 pontos em função da frequência dos comportamentos estudados ( 1 = nunca; 2 = quase nunca; 3 =algumas vezes; $4=$ quase sempre $;=$ sempre), e período de avaliação (últimos seis meses). Consistências interna encontradas neste estudo: alfa de Cronbach $=0,91$ (vitimização 0,89; agressão relacional 0,84; agressão direta 0,79 ).

Com o propósito de garantir a condição de alta frequência de agressão e/o vitimização que caracteriza o bullying, selecionamos como critérios para o envolvimento no bullying somente os casos que apresentaram nível alto (média mais metade do desvio padrão) nas dimensões: vitimização $(\mathrm{M}=14,88$; DP $=7,36)$, agressão relacional $(\mathrm{M}=11,88 ; \mathrm{DP}=5,47)$, agressão direta $(\mathrm{M}$ $=4,29 ; \mathrm{DP}=2,34)$. Sendo denominado como: $\mathrm{a}$ ) Agressor quem pontuou alto em Agressão relacional ou Agressão direta e não alto em Vitimização; b) Vitima quem pontuou alto em Vitimização e não alto em Agressão relacional e Agressão direta; c) Vitima-Agressora quem pontuou alto em Vitimização e alto em Agressão relacional ou Agressão direta.

\section{Análises estatísticas}

Dada a grande quantidade de variáveis e complexidade das interpretações, e com a finalidade de explorar tanto as implicações isoladas das variáveis estudadas (contextos de desenvolvimento, clima escolar, tipologia bullying, sexo, 
ano escolar, titularidade e territorialidade da escola), como a implicação de suas interações, sobre o bem-estar subjetivo, calculamos diversas análise de variância multivariada (MANOVAS):com variáveis separadas, com interações de duas variáveis, com interações de três variáveis, e finalmente com o conjunto de variáveis significativas resultantes, que é a MANOVA final que aqui será apresentada, tendo como variáveis dependentes a PWISC, SLSS, OLS. Seguindo as recomendações de Olson ${ }^{31}$, o traço Pillai-Barlett foi usado com um nível de significância não superior a $0,01^{32,33}$, dada a heterogeneidade existente na maioria dos resultados das matrizes de variância e covariância para as variáveis exploradas, de acordo com o teste de Levenee Box, respectivamente.

Para calcular as diferenças de médias de bem -estar subjetivo (escalas SLSS, PWI-SC, OLS) em função das variáveis: a) sociodemográficas sexo (menino/menina); ano escolar (6\%/7o) titularidade (público/privado), territorialidade da escola (urbano/rural); b) contextos de desenvolvimento (casa, escola, bairro); c) clima escolar (ambiente, regras, relacionamento), por nível baixo/alto (acima ou abaixo da média), utilizamos o teste $\mathrm{t}$ de Student (nível de significância $\mathrm{p}<0,05$ ). No caso das diferenças de médias de bem-estar em função da tipologia bullying (não envolvidos, vítimas, agressores, vítimas-agressores), utilizamos ANOVA de um fator com a prova Scheffé como contraste $(p<0,05)$. Resultados analisados através do uso do software Statistical Package for the Social Sciences (SPSS), versão 19.

\section{Aspectos éticos}

O projeto de pesquisa foi aprovado pelo Comitê de Ética em Pesquisa da Universidade de Fortaleza. Após autorizações institucionais no âmbito da Educação e, anterior às administrações dos questionários, foram necessárias as assinaturas do Termo de Concordância Institucional (gestores das escolas), Termo de Consentimento Livre e Esclarecido (responsáveis legais) e Termo de Assentimento Livre e Esclarecido (participantes). Os questionários foram respondidos de maneira anônima e os resultados são publicados de forma a garantir o anonimato dos participantes e das escolas.

\section{Resultados}

Serão apresentados os valores dos estatísticos ( $F$ e $p$ ) dos resultados significativos relativos às Ta- belas 1, 2, 3 e 4 (que por motivo de espaço não se encontram em ditas tabelas). Os relacionados à Tabela 5 devem ser consultados na mesma.

Como resultado dos critérios adotados para envolvimento no bullying temos que: $13,5 \%(N=$ 116) dos participantes do estudo foram classificados como agressores; $8,6 \%(N=74)$ como vítimas; $16,6 \%(N=143)$ como vítimas-agressoras e $61,3 \%(N=528)$ como não envolvidos.

Com relação às implicações das variáveis estudadas no bem-estar, a tabela 1 mostra os resultados da MANOVA final, com o conjunto de variáveis significativas (variáveis isoladas, interações de duas e de três variáveis). Os resultados indicam que a variável tipologiabullying é a que mais impacto exerce sobre o bem-estar, seja isoladamente ou em interação com as variáveis analisadas.

Em todas as formas de interação: Tipologiabullying\&TitularidadeぬAnoescolar (público/ $6^{\circ} \mathrm{a}-$ no $\mathrm{F}=94,78$-PWI-SC, $\mathrm{F}=131,91-\mathrm{OLS}, \mathrm{F}=$ 41,16-SLSS; público/7ªno $\mathrm{F}=102,07-\mathrm{PWI}-\mathrm{SC}$, $\mathrm{F}=58,94-\mathrm{OLS}, \mathrm{F}=33,02-\mathrm{SLSS} ;$ privado/7\%ano $\mathrm{F}=27,12-\mathrm{PWI}-\mathrm{SC}, \mathrm{F}=33,59-\mathrm{OLS}, \mathrm{F}=28,52$ SLSS, $\mathrm{p}<0,001)$; TipologiabullyingécontextoEscola\&Sexo (alto/menino $\mathrm{F}=63,60$; alto/menina $\mathrm{F}$ $=59,17$; baixo/menino $\mathrm{F}=27,57$; baixo/menina $\mathrm{F}=64,54, \mathrm{p}<0,001) ;$ Tipologiabullying \& Territorialidade (urbano $\mathrm{F}=128,88$, rural $\mathrm{F}=64,80, \mathrm{p}$ $<0,001$ ); TipologiabullyingécontextoBairro (alto $\mathrm{F}=98,30$, baixo $\mathrm{F}=89,72, \mathrm{p}<0,001$ ); TipologiabullyingécontextoCasa (alto $\mathrm{F}=79,05$, baixo $\mathrm{F}=$ $16,88, \mathrm{p}<0,001)$; os resultados indicam que os envolvidos (vítimas, agressores e vítimas-agressores) apresentam médias baixas de bem-estar, comparados aos não envolvidos, exceto entre vítimas e não envolvidos de $7^{\circ}$ ano de escolas privadas na OLS; entre envolvidos e não envolvidos de $6^{\circ}$ ano de escolas privadas na PWI-SC, OLS e SLSS e entre vítimas-agressores e não envolvidos na SLSS que as diferenças não são significativas. As vítimas apresentaram as piores médias, exceto dentro dos grupos com alto contexto bairro (vítimas-agressores) e os de $7{ }^{\circ}$ ano de escolas privadas (agressores) na OLS e SLSS (Tabelas 2 e 3).

Em relação às diferenças entre os envolvidos, nas interações TipologiaBullying\&TitularidadeEscola\&Anoescolar existem diferenças na PWI -SC e SLSS entre os de escolas públicas, de $6^{\circ}$ ano (vítimas e vítimas-agressoras $\mathrm{p}=, 046$ e $\mathrm{p}=0,30$ ) e entre os de $7^{\circ}$ ano (vítimas e agressores $\mathrm{p}=, 001$ e $\mathrm{p}<0,001$; vítimas e vítimas-agressoras $\mathrm{p}=, 006$ e $\mathrm{p}<0,001)$. E entre os alunos de escolas privadas de $7^{\circ}$ (vítimas e agressores $\mathrm{p}=, 018$; vítimas-agressoras e agressores $\mathrm{p}=, 046$ na OLS (Tabela 2). 


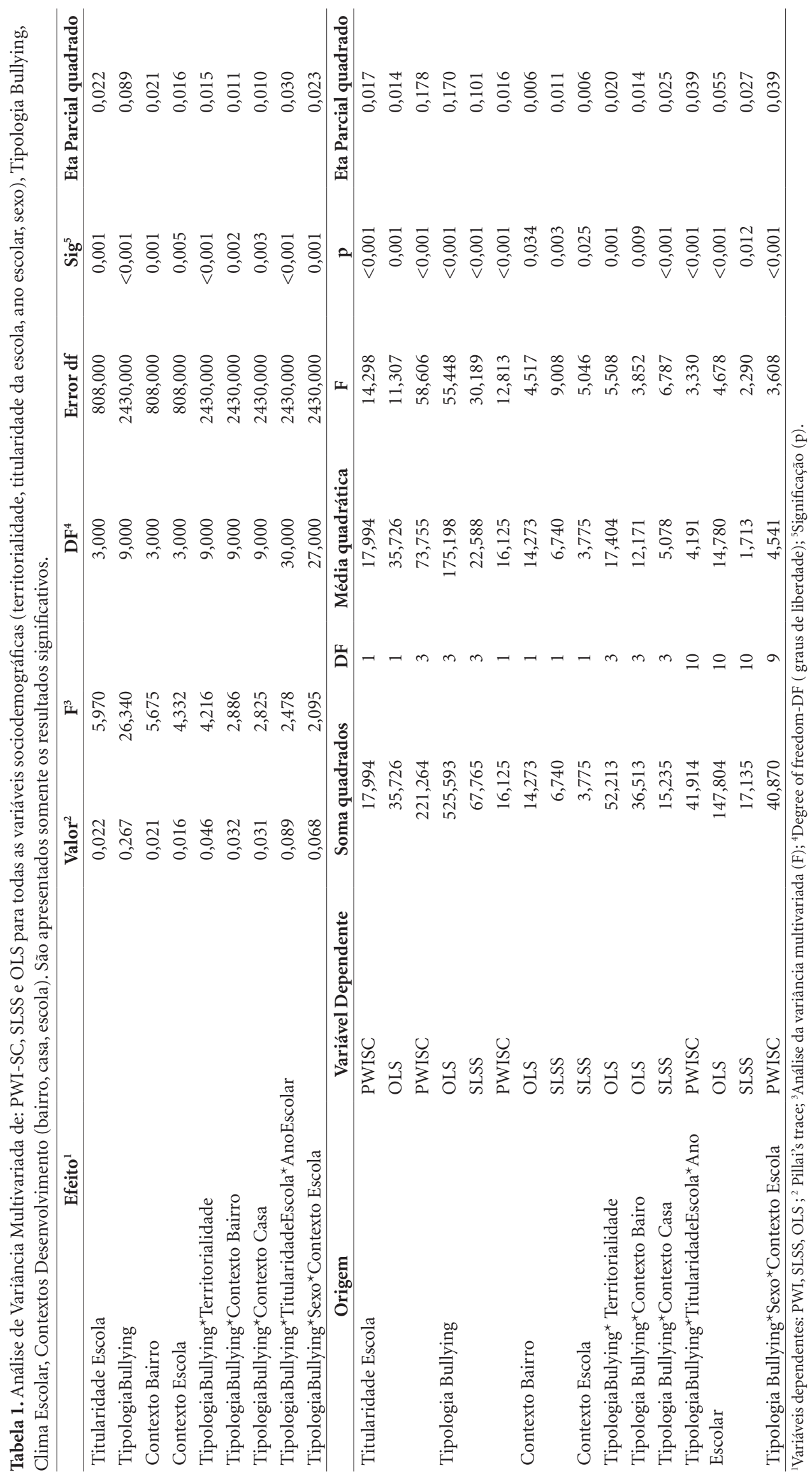




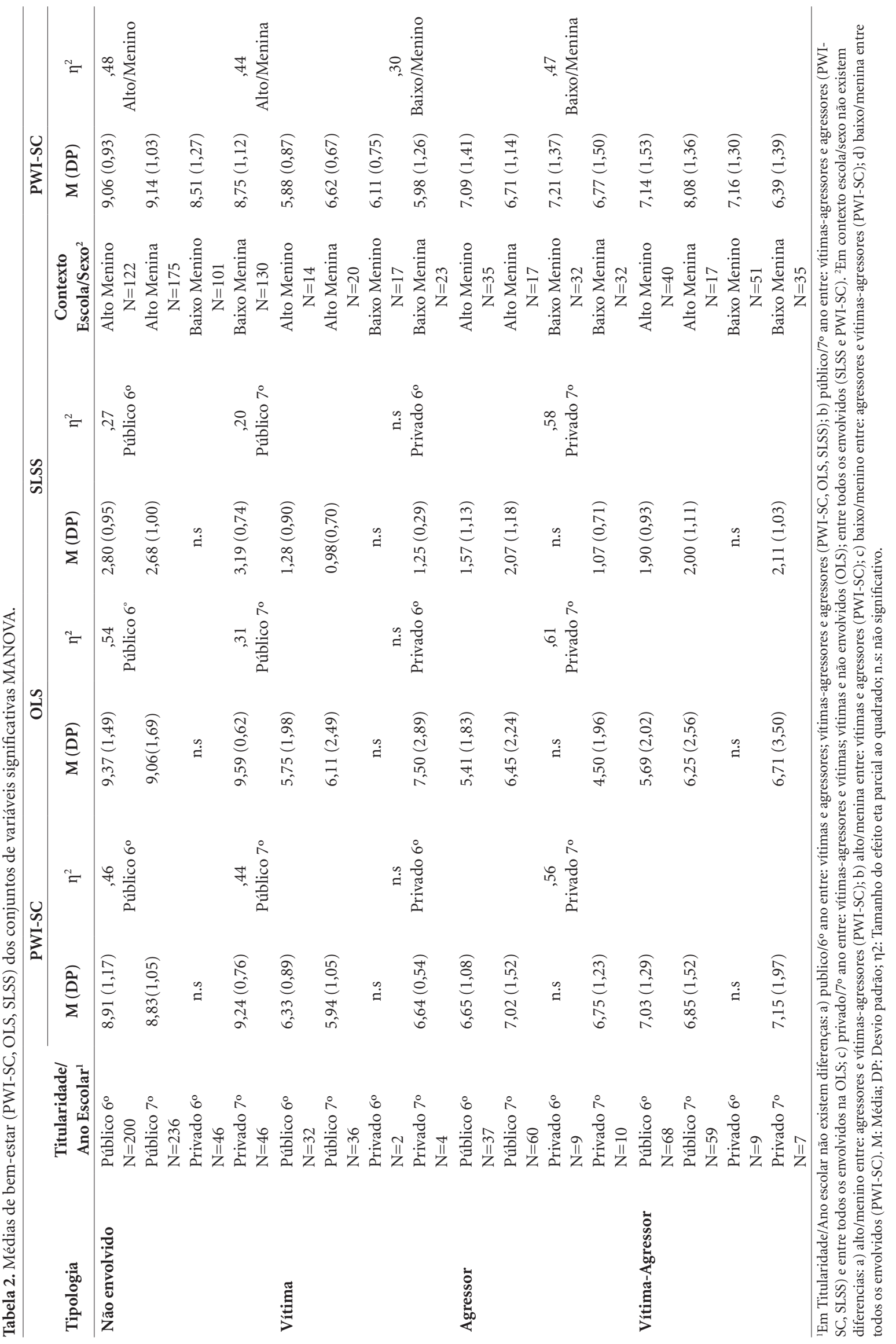




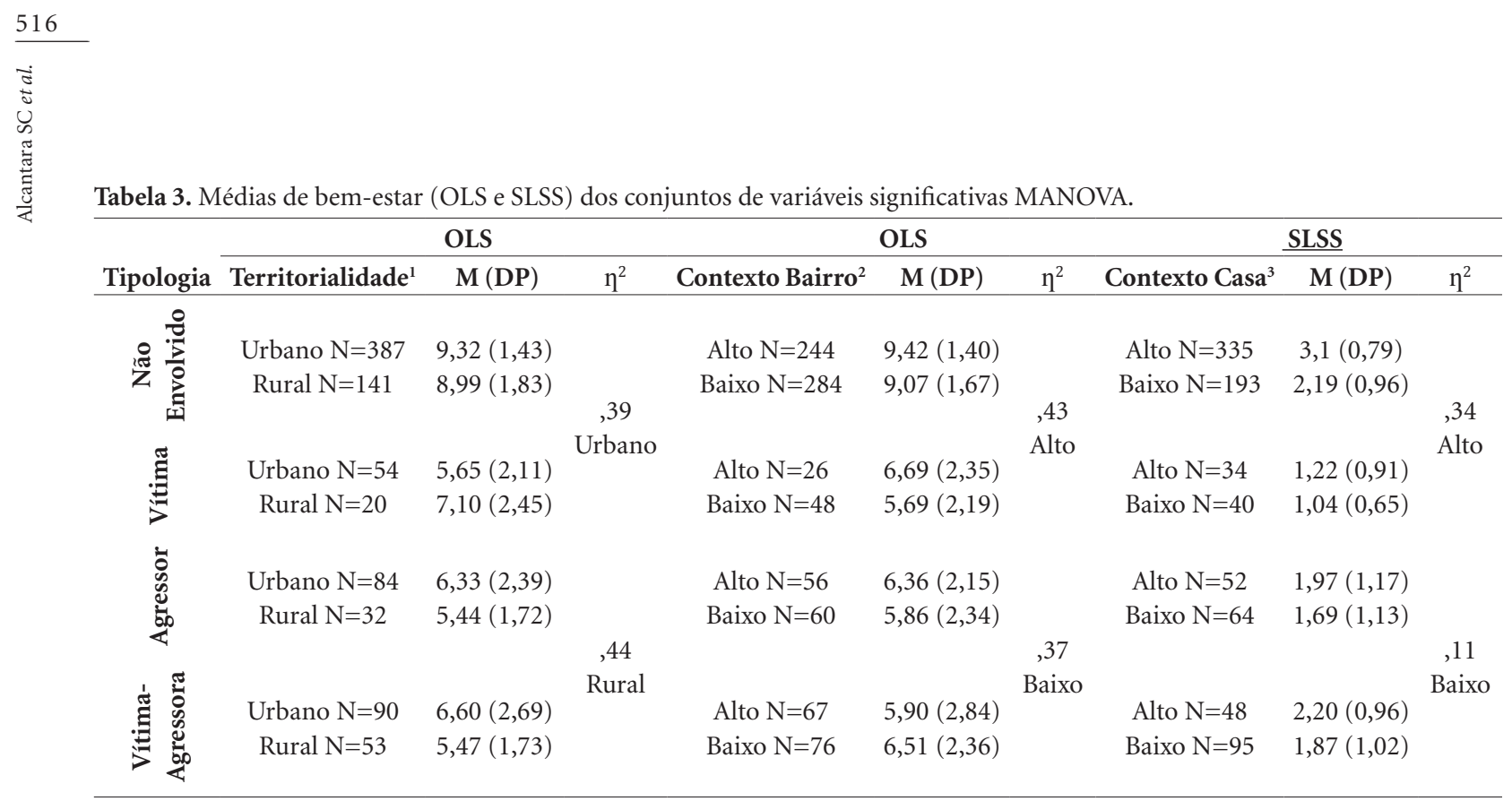

${ }^{1}$ Em Territorialidade urbana e rural não existem diferenças entre os envolvidos. ${ }^{2}$ Em contexto-bairro alto e baixo não existem diferenças entre envolvidos. ${ }^{3}$ Em contexto casa alto e baixo não existem diferenças entre agressores e vítimas-agressores; e em contexto casa baixo entre vítimasagressores e não envolvidos. M: Média; DP: Desvio padrão; $\eta^{2}$ :Tamanho do efeito eta parcial ao quadrado.

Tabela 4. Médias de Bem-Estar (SLSS, PWI-SC, OLS) em função da Tipologia Bullying (Vítima, Agressor, VítimaAgressora e Não Envolvido) ANOVA.

\begin{tabular}{|c|c|c|c|c|c|c|c|c|c|}
\hline \multirow[b]{2}{*}{ Tipologia } & \multicolumn{3}{|c|}{ SLSS } & \multicolumn{3}{|c|}{ PWI-SC } & \multicolumn{3}{|c|}{ OLS } \\
\hline & $\mathbf{M}$ & DP & $\eta^{2}$ & M & DP & $\eta^{2}$ & $\mathbf{M}$ & DP & $\eta^{2}$ \\
\hline 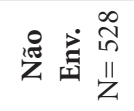 & 2,80 & 0,97 & & 8,91 & 1,10 & & 9,23 & 1,56 & \\
\hline$\stackrel{\Xi}{\stackrel{N}{N}}$ & 1,12 & 0,78 & & 6,16 & 0,97 & & 6,04 & 2,28 & \\
\hline 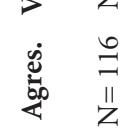 & 1,82 & 1,15 & .24 & 6,98 & 1,39 & .42 & 6,09 & 2,25 & .39 \\
\hline 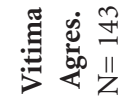 & 1,98 & 1,00 & & 7,08 & 1,47 & & 6,18 & 2,43 & \\
\hline
\end{tabular}

Diferenças significativas entre todos os grupos exceto entre: agressores e vítimas-agressores (PWISC, SLSS); entre envolvidos (OLS). Não Env.: Não envolvido; Agres.: Agressor; Vítima--Agres.: Vítima-agressor. M: Média; DP: desvio padrão; $\eta^{2}$ : Tamanho do efeito (eta) parcial ao quadrado. vítimas-agressoras $\mathrm{p}=, 001$, agressoras e vítimas -agressoras $p=, 003)$. Não existindo diferenças entre meninas envolvidas com baixo nível Contexto-Escola (Tabela 2).

Já nas interações TipologiaBullying\&Territorialidade e TipologiaBullying\&Contexto-Bairro não existem diferenças entre os envolvidos (Tabela 3 ).

Enquanto que em função da interação Tipologiabullying\&contextoCasa, existem diferenças entre os envolvidos com alto ou com baixo Contexo-Casa (agressores e vítimas, $\mathrm{p}=, 002$ e $\mathrm{p}=$ ,012; vítimas e vítimas-agressores $\mathrm{p}<0,001 \mathrm{e} \mathrm{p}<$ 0,001 ,sendo as vítimas as que apresentam as menores médias de bem-estar (Tabela 3).

Com relação a diferenças de médias de Bem-estar, os resultados da ANOVA (tabela 4) indicam que os envolvidos em bullying (vítimas, agressores e vítimas-agressoras) apresentaram médias baixas de bem-estar comparados aos não envolvidos $(\mathrm{F}=91,161-S L S S, \mathrm{~F}=210,65-\mathrm{PWI}-\mathrm{SC}, \mathrm{F}$ $=183,70$-OLS, $\mathrm{p}<0,001)$. Entre os envolvidos, também existem diferenças nas escalas PWI-SC e SLSS (vítimas e agressores $\mathrm{p}<0,001$, vítimas e vítimas-agressoras $\mathrm{p}<0,001)$. Não existindo diferenças de bem-estar entre os envolvidos na OLS.

Os resultados da prova $t$ de Student (Tabela $5)$ indicam que em função tanto dos contextos de desenvolvimento (casa, escola, bairro) como do agressores e vítimas $\mathrm{p}=, 041$ e $\mathrm{p}=, 012$ ) e entr meninas com alto Contexto-Escola (vítimas e 
Tabela 5. Médias dos três indicadores de Bem-Estar (SLSS, PWI-SC, OLS) em função das percepções dos Contextos de Desenvolvimento (Casa, Escola, Bairro); do Clima Escolar (Ambiente, Regras, Relacionamento); do sexo, da titularidade, da territorialidade da escola e do ano escolar, considerando toda a amostra.

\begin{tabular}{|c|c|c|c|c|c|c|c|c|c|c|c|c|c|}
\hline & & \multicolumn{4}{|c|}{ SLSS } & \multicolumn{4}{|c|}{ PWI-SC } & \multicolumn{4}{|c|}{ OLS } \\
\hline & & M & DP & $\mathbf{t}$ & d & M & DP & $\mathbf{t}$ & d & M & DP & $t$ & d \\
\hline \multirow{2}{*}{ שू } & Baixo $\mathrm{N}=412$ & 1,93 & 1,05 & $-12,46$ & 0,82 & 7,65 & 1,66 & $-8,63$ & 0,58 & 7,47 & 2,61 & $-6,74$ & 0,45 \\
\hline & Alto $\mathrm{N}=498$ & 2,80 & 1,06 & \multicolumn{2}{|c|}{$\mathrm{p}<0,001$} & 8,54 & 1,40 & \multicolumn{2}{|c|}{$\mathrm{p}<0,001$} & 8,54 & 2,13 & \multicolumn{2}{|c|}{$\mathrm{p}<0,001$} \\
\hline \multirow{2}{*}{ 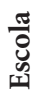 } & Baixo $\mathrm{N}=448$ & 2,09 & 1,08 & $-8,68$ & 0,57 & 7,81 & 1,62 & $-6,20$ & 0,41 & 7,79 & 2,48 & $-3,26$ & 0,22 \\
\hline & Alto $\mathrm{N}=462$ & 2,72 & 1,11 & \multicolumn{2}{|c|}{$\mathrm{p}<0,001$} & 8,45 & 1,49 & \multicolumn{2}{|c|}{$\mathrm{p}<0,001$} & 8,31 & 2,33 & \multicolumn{2}{|c|}{$\mathrm{p}=0,001$} \\
\hline \multirow{4}{*}{ 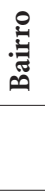 } & Baixo $\mathrm{N}=495$ & 2,15 & 1,12 & $-7,50$ & 0,50 & 7,95 & 1,62 & $-3,84$ & 0,20 & 7,92 & 2,44 & $-2,02$ & 0,14 \\
\hline & Alto $\mathrm{N}=415$ & 2,70 & 1,08 & & 0,001 & 8,27 & 1,57 & & 0,001 & 8.25 & 2.36 & & 0,044 \\
\hline & & \multicolumn{4}{|c|}{ SLSS } & \multicolumn{4}{|c|}{ PWI-SC } & \multicolumn{4}{|c|}{ OLS } \\
\hline & & $\mathbf{M}$ & DP & $\mathbf{t}$ & d & $\mathbf{M}$ & DP & $\mathbf{t}$ & d & $\mathbf{M}$ & DP & $\mathbf{t}$ & d \\
\hline \multirow{2}{*}{ 荌 } & Baixo $\mathrm{N}=399$ & 2,16 & 1,12 & $-5,96$ & 0,39 & 7,76 & 1,62 & $-6,25$ & 0,42 & 7,63 & 2,56 & $-4,64$ & 0,31 \\
\hline & Alto $\mathrm{N}=511$ & 2,60 & 1,12 & \multicolumn{2}{|c|}{$\mathrm{p}<0,001$} & 8,42 & 1,50 & \multicolumn{2}{|c|}{$\mathrm{p}<0,001$} & 8,39 & 2,24 & \multicolumn{2}{|c|}{$\mathrm{p}<0,001$} \\
\hline \multirow{4}{*}{ 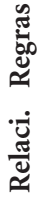 } & Baixo $N=440$ & 2,17 & 1,12 & $-6,24$ & 0,41 & 7,85 & 1,62 & $-5,33$ & 0,35 & 7,63 & 2,58 & $-5,20$ & 0,35 \\
\hline & Alto $\mathrm{N}=470$ & 2,63 & 1,11 & \multicolumn{2}{|c|}{$\mathrm{p}<0,001$} & 8,40 & 1,51 & \multicolumn{2}{|c|}{$\mathrm{p}<0,001$} & 8,46 & 2,18 & \multicolumn{2}{|c|}{$\mathrm{p}<0,001$} \\
\hline & Baixo $\mathrm{N}=439$ & 2,18 & 1,14 & $-6,03$ & 0,39 & 7,84 & 1,62 & $-5,44$ & 0,36 & 7,66 & 2,58 & \multirow{2}{*}{\multicolumn{2}{|c|}{$\begin{array}{r}-4,83 \\
\mathrm{p}<0,001\end{array}$}} \\
\hline & Alto $\mathrm{N}=471$ & 2,62 & 1,09 & \multicolumn{2}{|c|}{$\mathrm{p}<0,001$} & 8,40 & 1,50 & \multicolumn{2}{|c|}{$\mathrm{p}<0,001$} & 8,43 & 2,19 & & \\
\hline & & \multicolumn{4}{|c|}{ SLSS } & \multicolumn{4}{|c|}{ PWI-SC } & & $\mathbf{O}$ & & \\
\hline & & M & DP & $\mathbf{t}$ & d & $\mathbf{M}$ & DP & $\mathbf{t}$ & d & M & DP & $\mathbf{t}$ & d \\
\hline 8 & Menino $\mathrm{N}=436$ & 2,49 & 1,13 & 2,16 & 0,14 & 8,01 & 1,54 & $-2,23$ & 0,14 & 7,87 & 2,39 & $-2,21$ & 0,04 \\
\hline$\infty$ & Menina $\mathrm{N}=474$ & 2,33 & 1,14 & $\mathrm{p}=$ & ,031 & 8,24 & 1,62 & & 0,026 & 8,23 & 12,43 & & 0,027 \\
\hline है & Privada $N=147$ & 2,73 & 1,11 & $-3,77$ & 0,35 & 8,65 & 1,38 & $-4,86$ & 0,41 & 8,68 & 2,10 & $-3,82$ & 0,32 \\
\hline 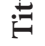 & Pública N=763 & 2,34 & 1,13 & $\mathrm{p}<$ &, 001 & 8,03 & 1,60 & & 0,001 & 7,94 & 2,45 & & 0,001 \\
\hline$\stackrel{0}{=}$ & Urbana $\mathrm{N}=651$ & 2,41 & 1,16 & 0,285 & 0,01 & 8,19 & 1,58 & 1,85 & 0,13 & 8,20 & 2,39 & 2,88 & 0,21 \\
\hline$\stackrel{\vec{\omega}}{\oplus}$ & Rural N=259 & 2,39 & 1,09 & n.s & & 7,98 & 1,59 & n.s & & 7,69 & 2,45 & & 0,004 \\
\hline 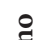 & $6^{\circ}$ Ano $\mathrm{N}=433$ & 2,43 & 1,13 & 0,767 & 0,04 & 8,20 & 1,58 & 1,21 & 0,08 & 8,07 & 2,44 & 0,130 & 0,01 \\
\hline$<$ & $7^{\circ}$ Ano $\mathrm{N}=477$ & 2,38 & 1,14 & n.s & & 8,07 & 1,58 & $\mathrm{n} . \mathrm{s}$ & & 8,05 & 2,39 & n.s & \\
\hline
\end{tabular}

M: média; DP: desvio padrão; t: prova t de Student; d: medida do tamanho do efeito de Cohen; n.s: não significativo; Ambi.:AmbienteEscolar; Relaci.: Relacionamento-Escola; Titular: Titularidade; Territo: Territorialidade.

clima escolar (ambiente-escolar, regras, relacionamento-escolar) os alunos que apresentaram nível baixo pontuaram médias mais infriores em todas as escalas de bem-estar, comparados aos que apresentaram nível alto nestas dimensões. Em função do gênero, as meninas mostram médias mais elevadas que os meninos nas escalas PWI-SC $e$ OLS. Já os resultados relacionados a titularidade da escola apontam que os alunos de escolas públicas apresentam pontuações mais baixa nas escalas de bem-estar (SLSS, PWI-SC, OLS), comparados com os das privadas. E, por territorialidade, alunos das escolas rurais pontuam mais baixo que os das urbanas na OLS. Não observando diferenças em função do ano escolar (Tabela 5).

\section{Discussão}

Observamos que todas as variáveis estudadas apresentam diferenças significativas seja na prova t de Student, ANOVA ou na MANOVA, ainda que nesta última o critério de significância adotado tenha sido mais restritivo (não superior a 0,01).

Em função da interação TipologiaBullying\&TitularidadeEscola\&Anoescolar, os resultados indicam que entre os alunos do $7^{\circ}$ ano das escolas públicas são as vítimas as que obtêm as médias mais baixas de bem-estar na PWI-SC e SLSS, comparadas tanto aos não-envolvidos, como aos agressores e as vitimas-agressoras. Diferentemente dos resultados do $7^{\circ}$ ano de escolas privadas em 
que são os agressores os que apresentam níveis mais baixos de bem-estar na OLS e SLSS, quando comparados aos não-envolvidos e na OLS quando comparados as vítimas e as vitimas-agressores. $\mathrm{O}$ que pode sugerir que o contexto social onde ocorre a violência (escolas públicas/privadas) interfere na vivência de ser vítima ou agressor e, por conseguinte, na forma como afeta o bem-estar dos participantes. Parecendo ser o comportamento de agressor menos tolerável nas escolas privadas. Em relação às diferenças entre envolvidos das escolas públicas, tanto o grupo dos de $7^{\circ}$ como os de $6^{\circ}$ ano apresentam diferenças em bem-estar nas escalas PWI-SC e SLSS entre vítimas-agressoras e vítimas, tendo estas últimas, as piores médias em ambos os anos escolares. Em todas as formas de interação entre as variáveis $T i$ pologiaBullying\&TitularidadeEscola\&Anoescolar, os alunos envolvidos em bullying apresentam médias mais baixas de bem-estar em todas as escalas, comparados aos não envolvidos. O que permite concluir em consonância com estudos anteriores que a violência física e outras formas de bullying aumentam a vulnerabilidade/risco frente aos determinantes de saúde $\mathrm{e}^{34}$, afetando o bem-estar e a qualidade de vida de todos os envolvidos: vítima, agressor e vítima-agressora ${ }^{1,5,19}$.

Considerando a interação Tipologiabullying\&Sexo\&Contexto-Escola, as vitimas sejam com baixo ou alto nível Contexto-Escola, meninos ou meninas obtiveram as médias mais baixas de bem - estar (PWI-SC), comparadas aos não-envolvidos com alto ou baixo Contexto-Escola, meninos ou meninas. Entre as meninas envolvidas em bullying com alto nível Contexto-Escola existem diferenças entre vítimas-agressoras e vítimas, sendo as últimas as que apresentam médias de bem-estar mais baixas (PWI-SC). No caso dos meninos envolvidos seja com alto ou baixo nível Contexto-Escola são as vítimas que apresentam médias mais baixa de bem-estar, quando comparadas aos agressores e as vítimas-agressoras. Estes resultados parecem indicar que, independente do sexo (menina ou menino) e do nível de percepção do Contexto-Escola (baixo ou alto), ser vítima de bullying se configura como um dos principais fatores de risco associado à obtenção de níveis baixos de bem-estar. Além disso, os resultados indicam que em todas as formas de interação entre as variáveis, os alunos envolvidos em bullying apresentam médias mais baixas de bem-estar, comparadas aos não envolvidos. Nesta mesma direção, estudo com escolares canadenses, realizado por Guhn et al. ${ }^{6}$, aponta que a vitimização está associada com baixa satisfação com a vida. Estudo comparativo entre Espanha e França indica que os escolares que sofrem violência são os que se sentem mais inseguros na escola e os que têm a percepção mais negativa do Clima Escolar ${ }^{35}$.

Em função da interação TipologiaBullyingeTerritorialidade observamos que entre os envolvidos, as vítimas da urbana e os agressores da rural apresentam as piores médias na OLS, comparados aos não envolvidos. O que parece indicar, novamente, que a vivência de ser vítima ou agressor e, por conseguinte, a forma como afeta o bem-estar tem relação com o contexto social onde ocorrem tais experiências (urbano/rural). Nos contextos urbanos, talvez, o comportamento mais esperado e positivamente valorizado entre os iguais seja o de ser agressor, diferentemente dos contextos rurais.

A interação entre as variáveis TipologiaBullyingerContexto-Bairro parece indicar que o fato de não se envolver em bullying e ter uma boa percepção do contexto onde se vive pode se configurar como fatores protetivos em relação a niveis mais elevados de bem-estar, uma vez que os não envolvidos com alto nível de Contexto-Bairro são os que apresentam as melhores médias, em consonância com estudos anteriores ${ }^{7,36}$. Dentro do grupo com baixo nível de Contexto-Bairro as vítimas, comparadas aos não envolvidos, são as que apresentam médias mais baixas de bem-estar. E dentro do grupo com alto nível Contexto-Bairro as vitimas-agressoras, quando comparados aos não envolvidos, são as que obtêm as piores médias na OLS. Parece ser que sentir-se ou não seguro afeta negativamente o bem-estar dos envolvidos. No caso das vítimas-agressoras que apresentam nível alto no Contexto-Bairro a segurança parece advir do grupo de iguais. Dentro do grupo o comportamento violento é sinônimo de popularidade, poder, fama, o que faz com que alguns adolescentes entrem ou sigam na dinâmica da agressão/vitimização como ferramenta para lograr tais objetivos ${ }^{4,5}$.

Nesta mesma direção de análise, a interação TipologiaBullyingぬ Contexto-Casa, aponta que as vítimas sejam com baixa ou alta percepção do Contexto-Casa apresentam as piores médias de bem-estar na SLSS, comparadas tanto aos não-envolvidos, como aos agressores e as vítimas-agressoras. Novamente, dentro da dinâmica do bullying, as vitimas apresentam as médias mais baixas de bem-estar ainda que dentro do grupo com alta percepção do Contexto-Casa. O que pode indicar que o fato de não se envolver em bullying, independente de pertencer ao grupo de alto ou baixo nível Contexto-Casa, já é um fator protetivo 
a melhores níveis de bem-estar. Ao contrário, o fato de estar envolvido acrescido à baixa percepção do Contexto-Casa, pode configurar-se como fatores de maior risco associados à diminuição do bem-estar. Resultados estes na mesma direção de estudos anteriores ${ }^{7}$.

A TipologiaBullying representa o fator de risco/proteção mais associado ao bem-estar subjetivo, sendo os envolvidos em comportamentos de bullying os que obtêm as menores médias de bem-estar em todas as escalas, comparados com os não envolvidos, em consonância com estudos anteriores $^{1,5,19}$. Dentro do grupo de envolvidos, as vítimas apresentam as piores médias na PWI -SC, SLSS, em consonância com estudo realizado por Navarro et al. ${ }^{1}$ e em divergência com estudos que indicam que são as vítimas-agressoras as que apresentam médias mais baixas em satisfação com a vida ${ }^{5,19}$.

A percepção dos contextos de desenvolvimento casa, escola, bairro também representa papel relevante para definir o nível de bem-estar, sendo o nível baixo associado a médias menores de bem-estar. Tais resultados estão na mesma direção dos obtidos por Lee and Yoo ${ }^{15}$, em estudo comparativo internacional, que aponta que os contextos família, escola, comunidade afetam significativamente os níveis de bem-estar de crianças/adolescentes. Quando analisamos o Contexto-Escola, especificamente os elementos do Clima Escolar, observamos que baixos níveis de percepção do ambiente-escola, regras, relacionamento-escolar estão associados a baixos niveis de bem-estar em todas as escalas estudadas, sendo estes resultados congruentes com estudos anteriores ${ }^{7,8}$. Tais resultados indicam que a escola, enquanto ambiente de ensino/aprendizagem e socialização representa um contexto significativo ao desenvolvimento psicossocial saudável, sendo necessário fomentar um clima escolar positivo que favoreça a convivência entre todos os atores sociais e, por conseguinte, resulte em melhores níveis de bem-estar e menores níveis de violência entre iguais, e viceversa $^{8-10}$.

Todas as variáveis estudadas se destacam como fatores relevantes que podem informar da vulnerabilidade/risco da criança/adolescente envolver-se na dinâmica do bullying. Ou, ao contrário, o envolvimento no bullying pode apresentar-se como fator de vulnerabilidade/risco frente a pontuar baixos níveis de percepção dos contextos de desenvolvimento e do clima escolar, impactando, em ambos os casos, de forma negativa no bem-estar de todos os envolvidos. No extremo oposto, temos as crianças/adolescentes não envolvidos que apresentam as melhores médias em todas as variáveis estudadas, inclusive em bem-estar subjetivo, em consonância com estudos anteriores $^{1,5,19}$.

Em relação às variáveis sociodemográficas, em função do sexo os resultados são inconclusos, uma vez que dependem da escala utilizada; estando em consonância com a literatura ${ }^{37}$. De forma geral, ainda que se obtenha, em alguns casos, os mesmos resultados com as três escalas de bem -estar, em outros casos os resultados diferem em função da escala utilizada, o que indica, em congruência com a literatura, que não estão medindo exatamente os mesmos aspectos ${ }^{12}$. Além disso, os resultados indicam que ser aluno de escola pública e/ou morar/estudar em zona rural estar associado à obtenção de níveis mais baixos de bem-estar, em discordância com o estudo espanhol realizado por Casas et al..$^{37}$, que indica médias mais altas de bem-estar em alunos de escolas públicas e de zonas semi-urbanas. Estes dados do contexto brasileiro podem indicar as iniquidades sociais existentes tanto entre classes (uma vez que os alunos de escolas públicas advêm de famílias empobrecidas), como entre contextos territoriais (escasso investimento público nas zonas rurais), o que desemboca na diminuição do bem-estar das crianças/adolescentes.

Todos os resultados nos ratificam a importância de estudar os fatores de risco/proteção associados ao bem-estar subjetivo, desde a percepção que crianças e adolescentes têm acerca do fenômeno do bullying e dos âmbitos de desenvolvimento casa, escola e bairro. Com atenção à criação e manutenção de entornos e ambientes saudáveis ao desenvolvimento psicossocial de crianças e adolescentes, pautados na promoção de saúde e qualidade de vida, como resultante de um processo de transformação dos fatores determinantes sociais de saúde e de bem-estar ${ }^{38}$.

Uma das limitações desta investigação tem relação com a falta de controle de confundimento por outras variáveis que podem estar relacionadas às variáveis estudadas e influenciar o bem-estar. Além disso, a metodologia transversal não permite estabelecer relações de tipo causal, nem conhecer a evolução dos comportamentos de vitimização e agressão e/ou o nível de bem-estar subjetivo dos participantes com o avanço do tempo; para estabelecer relações causais seria necessário desenvolver uma pesquisa longitudinal com as mesmas variáveis aqui estudadas. Futuras pesquisas poderiam orientar-se a conhecer como o bullying incide no bem-estar a partir de estudos longitudinais e/ou qualitativos. Por último, 
seria interessante pesquisas comparativas em nível nacional e internacional explorando como o bullying incide no bem-estar segundo as características de distintos contextos e segundo diferenças socioeconômicas e culturais.

\section{Colaboradores}

SC Alcantara participou de todas as etapas do estudo. M González-Carrasco contribuiu com a interpretação dos dados, revisão crítica e concepção final do artigo. C Montserrat, F Viñas-Poch e F Casas: contribuíram com revisão crítica e concepção final do artigo. DP Abreu: contribuiu com a revisão crítica do artigo.

\section{Agradecimentos}

À Coordenação de Aperfeiçoamento de Pessoal de Nível Superior- CAPES (Bolsa Doutorado Pleno no Exterior). 


\section{Referências}

1. Navarro R, Ruiz-Oliva R, Larrañaga E, Yubero S. The impact of cyberbullying and social bullying on optimism, global and school-related happiness and life satisfaction among 10-12-year-old schoolchildren. Apples Res Qual Life 2015; 10(1):15-36.

2. World Health Organization (WHO). Health Behaviour in School-aged Children (HBSC). International Report from the 2009/2010 survey. Social determinants of health and well-being among young people. [Internet]. 2012 [acessado 2015 Jul 20]. Disponível em: http://www. euro.who.int/_data/assets/pdf_file/0007/167425/E9 6444_part2_5.pdf

3. Olweus D. El acoso escolar: una revisión general. In: Serrano A, editor. Acoso y violencia en la escuela: como detectar, prevenir y resolver el bullying. Barcelona: Editorial Ariel; 2006. p. 152-260.

4. Moreno DR, Estévez EL, Murgui SP, Musitu GO. Reputación social y violencia relacional en adolescentes: el rol de la soledad, la autoestima y la satisfacción vital. Psicothema 2009; 21(4):537-542.

5. Povedano A, Estévez E, Martínez B, Monreal M-C. Un perfil psicosocial de adolescentes agresores y víctimas en la escuela: análisis de las diferencias de género. Rev Psicol Soc 2012; 21(2):169-182.

6. Guhn M, Schonert-Reichl K, Gadermann A, Hymel S, Hertzman C. A population study of victimization, relationships, and well-being in middle childhood. J Happiness Stud 2013; 14(5):1529-1541.

7. Jiménez TI, Lehalle H. La violencia escolar entre iguales en alumnos populares y rechazados. Psychosoc Interv 2012; 21(1):77-89.

8. Martínez BF, Moreno DR, Amador LV, Orford J. School victimization among adolescents: an analysis from an ecological perspective. Psychosoc Interv 2011; 2(20):149-160.

9. Lindstrom-Johnson S, Waasdorp TE, Debnam K, Bradshaw $\mathrm{CP}$. The role of bystander perceptions and school climate in influencing victims' responses to bullying: to retaliate or seek support? Brit J Criminol 2013; 2013:780460.

10. Martínez-Ferrer B, Povedano-Díaz A, Amador-Muñoz LV, Moreno-Ruiz D. Clima escolar, satisfacción con la vida y victimización en la escuela: un análisis del efecto moderador del género. An Psicol Spain 2012; 28(3):875882.

11. Espelage DL, Swearer SM. A social-ecological model for bullying prevention and intervention: understanding the impact of adults in the social ecology of youngsters. In: Jimerson SR, Swearer SM, Espelage DL, editores. Handbook of bullying in schools: An international perspective. New York: Routledge; 2010. p. 61-72.

12. Casas F, Bello A. Calidad de vida y bienestar infantil subjetivo en España ¿Qué afecta al bienestar de niños y niñas españoles de $1^{\circ}$ de ESO? Madrid: Unicef; 2012.

13. Barbosa AJ, Moreira PS. Escala de Clima Escolar. Juiz de Fora: Autores; 2009

14. Comisión sobre Determinantes Sociales de la SaludCDSS. Informe de la Secretaria A62/9 del 16 de marzo de 2009. [Internet] Ginebra: Organización Mundial de la Salud; 2009. [acessado 2015 Jul 20]. Disponível em: http://apps.who.int/gb/ebwha/pdf_files/A62/A62_ 9-sp.pdf
15. Lee BJ, Yoo MS. Family, school, and community correlates of children's subjective well-being: an international comparative study. Chid Ind Res 2015; 8(1):151175.

16. Elvas S, Moniz MJ. Sentimento de comunidade, qualidade e satisfação de vida. Anál. psicol. 2010; 28 (3):451464.

17. González M, Gras M, Mao S, Navarro D, Casas F, Aligué $\mathrm{M}$. Adolescent's perspective on their participation in the family context and its relationship with their subjective well-being. Child Ind Res 2015; 8(1):93-109.

18. Ronen T, Hamama L, Rosenbaum M, Mishely-Yarlap A. Subjective well-being in adolescence: the role of self-control, social support, age, gender, and familial crisis. J Happiness Stud 2014; 17(1):81-104.

19. Flaspohler P, Elfstrom J, Vanderzee K, Sink H. Stand by me: The effects of peer and teacher support in mitigating the impact of bullying on quality of life. Psychol Sch 2009; 46(7):636-649.

20. Rees G, Main G, editors. Children's views on their lives and well-being in 15 countries: An initial report on the Children's Worlds survey, 2013-14. New York: Children's Worlds Project; 2015.

21. Soares AHR, Martins AJ, Lopes MC, Britto JA, Oliveira, CQ, Moreira, MCN. Qualidade de vida de crianças e adolescentes: uma revisão bibliográfica. Cien Saude Colet 2011; 16(7):3197-3206.

22. Casas F, Alfaro J, Sarriera J, Bedin L, Grigoras B, Balttătescu S, Malo S, Sirlopú D. El bienestar subjetivo en la infancia: estudio de la comparabilidad de 3 escalas psicométricas en 4 países de habla latina. Psicoperspectivas 2015; 14(1):6-18.

23. Bedin L, Sarriera, J. Propriedades psicométricas das escalas de bem-estar: PWI, SWLS, BSLSS e CAS. Avaliação Psicológica 2014; 13(2):213-225.

24. Campbell A, Converse P, Rodgers W. The quality of American life: perceptions, evaluations and satisfactions. New York: Russel Sage Foundation; 1976.

25. Cummins R, Eckersley R, Pallant J, Van Vugt J, Misajon R. Developing a national index of subjective wellbeing: The Australian Unity Wellbeing Index. Social Indicators Research 2003; 64(2):159-190.

26. Diener E, Emmons R, Larsen R, Griffin S. The Satisfaction With Life Scale. Journal of Personality Assessment 1985; 49(1):71-75.

27. Schütz F, Sarriera J, Bedin L, Montserrat C. Subjective well-being of children in residential care: Comparison between children in institutional care and children living with their families. Psicoperspectivas 2015; 14(1):19-30

28. Veiga FH, Antunes J, Guerra TM, Moura HM, Fernandes L, Roque P. Clima de escola: Uma escala de avaliação (CLES). X Conferência Internacional de Avaliação Psicológica: Formas e Contextos; 2004; Portugal. p. 545550. Universidade do Minho, Instituto de Educação e Psicologia.

29. Cunha JM, Weber LN, Steiner P. Escala de vitimização e agressão entre pares (EVAP). In: Weber L, Dessen MA, editores. Pesquisando a Família- Instrumentos para Coleta e Análise de Dados. Curitiba, Brasil: Juruá; 2009. p. $16-62$. 
30. Orpinas P, Frankowski R. The aggression scale: A self-report measure of aggressive behavior for young adolescents. Journal of Early Adolescence 2001; 21(1):50-67.

31. Olson CL. Practical considerations in choosing a MANOVA test statistic: a rejoinder to Stevens. Psychol. Bull. 1979; 86(6):1350-1352.

32. Field A. Discovering Statistics Using SPSS. $3^{\text {th }}$ ed. Sussex: University of Sussex, SAGE; 2010.

33. Hair JF, Black WC, Babin BJ, Anderson R. Multivariate data analysis. $7^{\text {th }}$ ed. Upper Saddle River: Prentice Hall; 2009

34. Andrade SS, Yokota R, Sá N, Silva M, Araújo W, Mascarenhas MDM, Malta DC. Relação entre violência física, consumo de álcool e outras drogas e bullying entre adolescentes escolares brasileiros. Cad Saúde Pública 2012; 28(9):1725-1736

35. Blaya C, Debarbieux E, Ortega R, Rey R. Clima y violencia escolar: un estudio comparativo entre España y Francia. Rev Educ 2006; 339:293-315

36. Jiménez TI, Estévez E, Murgui S. Ambiente comunitario y actitud hacia la autoridad: relaciones con la calidad de las relaciones familiares y con la agresión hacia los iguales en adolescents. Anales de psicología 2014; 30(3):1086-1095

37. Casas F, Bello A, González M, Aligué M. Children’s subjective well-being measured using a composite index: what impacts Spanish first-year secondary education students' subjective well-being? Child Ind Res 2013; 6(3):433-460.

38. Ippolito-Shepherd J. Escolas promotoras de saúde - fortalecimento da iniciativa regional. Estratégias e linhas de ação 2003-2012. Washington: Organização Pan-americana de Saúde (OPAS); 2006

Artigo apresentado em 17/12/2015

Aprovado em 08/05/2017

Versão final apresentada em 10/05/2017 